\title{
Impact of Fibrosis-4 Index Prior to COVID-19 on Outcomes in Patients at Risk of Non-alcoholic Fatty Liver Disease
}

\author{
Mohamed A. Elfeki ${ }^{1,2,6}$. Julian Robles ${ }^{1,2} \cdot$ Zaheer Akhtar $^{1,2} \cdot$ Fauzia Ullah $^{1,2} \cdot$ Ice Ganapathiraju ${ }^{1,2} \cdot$ Calvin Tran $^{3,5}$. \\ Casey Inman ${ }^{3,5} \cdot$ Simon M. Collin ${ }^{4} \cdot$ Rossana Rosa $^{5}$
}

Received: 8 February 2021 / Accepted: 16 June 2021 / Published online: 26 June 2021

(c) The Author(s), under exclusive licence to Springer Science+Business Media, LLC, part of Springer Nature 2021

\begin{abstract}
Background Severity of disease and outcomes in patient with COVID-19 has been associated with several risk factors tied to the metabolic syndrome.

Aims We conducted a study with the objective of describing the association between the baseline Fibrosis-4 (FIB-4) index prior to SARS-CoV-2 infection and the severity of COVID-19 among patients at risk of non-alcoholic fatty liver disease (NAFLD).

Methods This was a retrospective cohort study of patients with at least two risk factors for metabolic syndrome diagnosed with COVID-19. The main exposure of interest was FIB-4 index prior to infection, categorized into three previously validated age-specific levels. The main outcomes of interest were disease requiring hospitalization and in-hospital mortality.

Results We included 373 patients [median age, 62 years; 194 male (52\%); median number of metabolic syndrome risk factors, 3]. The median FIB-4 index was 1.10 (interquartile range 0.78-1.61). In models adjusting for diabetes mellitus and chronic kidney disease, patients with intermediate FIB-4 index had 67\% higher odds of hospitalization compared to those in the low category \{odds ratio (OR) 1.67 [(95\% CI 1.06-2.64); $p=0.03]$ ] and patients with high FIB-4 index had higher odds of mortality compared to intermediate and low category with an OR 2.22 (95\% CI 1.20-4.12; $p=0.01)$. However, when we evaluated components of FIB-4 (age and AST/ALT ratio), we found that age alone was the best predictor of hospitalization and mortality.
\end{abstract}

Conclusions Among patients at risk of NAFLD with COVID-19 infection, elevated pre-infection FIB-4 index was associated with worsened clinical outcomes, but age was the strongest predictor.

Keywords SARS-CoV-2 $\cdot$ Metabolic syndrome $\cdot$ Liver fibrosis $\cdot$ Non-alcoholic steatohepatitis $\cdot$ Metabolic-associated fatty liver disease

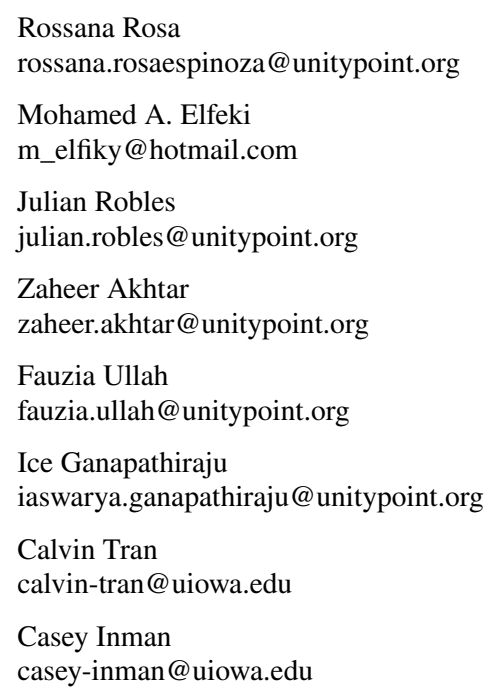

Simon M. Collin

simon.collin@phe.gov.uk

1 Internal Medicine Department, University of Iowa, Des Moines, IA, USA

2 Iowa Methodist Medical Center, UnityPoint Health-Des Moines, 1415 Woodland Avenue, Des Moines, IA 50309, USA

3 Carver College of Medicine, University of Iowa, Iowa City, IA, USA

4 National Infection Service, Public Health England, 61 Colindale Avenue, London NW9 5EQ, UK

5 Infectious Diseases Service, UnityPoint Health-Des Moines, 1221 Pleasant Street, Suite 300, Des Moines, IA 50309, USA

6 Present Address: Avera Medical Group Liver Disease, 1315 South Cliff Avenue, Suite 200-Plaza 3, Sioux Falls, SD, USA 


\section{Introduction}

Severe acute respiratory syndrome coronavirus 2 (SARS$\mathrm{CoV}-2$ ) and its corresponding disease, coronavirus disease (COVID-19) were initially described in Wuhan, China in December 2019 [1]. The rapid worldwide spread of SARSCoV-2 led the World Health Organization (WHO) to declare COVID-19 a global pandemic on March 11, 2020 [2]. The USA has been widely affected by SARS-CoV-2, with over 25 million cases and 425,000 deaths as of January 2021 [3].

Early during the COVID-19 pandemic, reports tied preexisting chronic medical comorbidities and advanced age to the severity of COVID-19 illness [4]. There is now a growing body of literature specifically describing the deleterious effects of pre-existing metabolic syndrome (MetS) risk factors (central obesity, diabetes mellitus [DM], hypertension, hypertriglyceridemia and low levels of high-density lipoproteins [HDL]) on the severity of COVID-19 illness [5-7]. In the USA, it is estimated that nearly a third of the population meets the definition for metabolic syndrome [8], thus potentially exacerbating the risks from COVID-19.

Non-alcoholic fatty liver disease (NAFLD), recently renamed as metabolic-associated fatty liver disease (MAFLD) $[9,10]$, is a quiescent disease with a slow asymptomatic progress for years before it declares itself clinically. Genetic, racial and environmental risk factors may contribute to acceleration of the disease course. The prognosis of NAFLD is primarily determined by the severity of liver fibrosis [11] Furthermore, patients with NAFLD have been noted to have a higher risk of liver injury when diagnosed with COVID-19 [12]. The Fibrosis-4 (FIB-4) index is a simple, well-validated point-of-care tool that is used to risk-stratify the degree of liver fibrosis among patients with suspected NAFLD $[13,14]$. It includes patient's age, alanine aminotransferase (ALT), aspartate aminotransferase (AST) and platelet count, which can be easily calculated by frontline providers.

In this study, we aimed to describe the relationship between intermediate or elevated FIB-4 prior to SARSCoV-2 infection and the severity of COVID-19 among patients at risk of NAFLD.

\section{Methods}

\section{Setting and Population}

This study was conducted among patients presenting to any UnityPoint clinic or hospital in the state of Iowa, USA, from March 15, 2020, to April 30, 2020, and diagnosed with COVID-19 by polymerase chain reaction (PCR) test. UnityPoint has a network of medical facilities throughout the state of Iowa which are divided in 7 geographical regions (Supplemental Fig. 1). The sample was limited to individuals at risk of NAFLD (defined as the presence of $\geq 2$ MetS risk factors in absence of significant alcohol use). Study participants were identified from a microbiology central database. Baseline clinical and demographic information was obtained from the centralized electronic medical record. We included patients with complete data for AST, ALT, platelets, HDL and triglycerides at a minimum of 2 weeks and maximum of 3 years prior to COVID-19 diagnosis in order to ensure that these values reflected a pre-COVID-19 diagnosis baseline. Exclusion criteria were age $<35$ years, significant alcohol use and history of underlying chronic liver disease due to viral hepatitis, alcohol use disorder, autoimmune diseases or hereditary disorders.

\section{Design, Outcome and Exposures}

This was a retrospective cohort study. The main outcomes of interest were disease requiring hospitalization and inhospital mortality (including discharge to hospice). The main exposure of interest was FIB-4 index categorized into three previously validated age-specific levels [14]. For patients between age 35 years old and less than 65 , cutoffs were $<1.30,1.30-2.67$ and $>2.67$ for low, intermediate and high risk of fibrosis, respectively. For patients 65 years and older, the cutoffs used were $<2,2-2.67$ and $>2.67$ for low, intermediate and high risk of fibrosis, respectively.

Data regarding comorbidities of interest were abstracted from the electronic medical record and included gender, body mass index (BMI), hyperlipidemia, hypertriglyceridemia, hypertension, diabetes mellitus, obstructive sleep apnea (OSA), chronic kidney disease (CKD), coronary artery disease (CAD), and hypothyroidism. Since data regarding abdominal circumference were not available, a formula for conversion of BMI to abdominal circumference was used in order to estimate central obesity [15].

\section{Statistical Analysis}

Variables were described using proportions and medians as appropriate. Effects of FIB-4 index on odds of hospitalization and in-hospital mortality were estimated using logistic regression with clustered-robust standard errors to account for clustering by different geographic regions. Direct acyclic graphs were constructed to identify relationships between variables and to inform selection of variables as potential confounders (Supplemental Fig. 2) [16]. Since age, platelet count, AST and ALT are included in the FIB-4 index it was decided a priori not to adjust for the individual patient values in our primary model. We also investigated associations of age and AST/ALT ratio with our outcomes by fitting models with these variables and comparing c-statistics between 


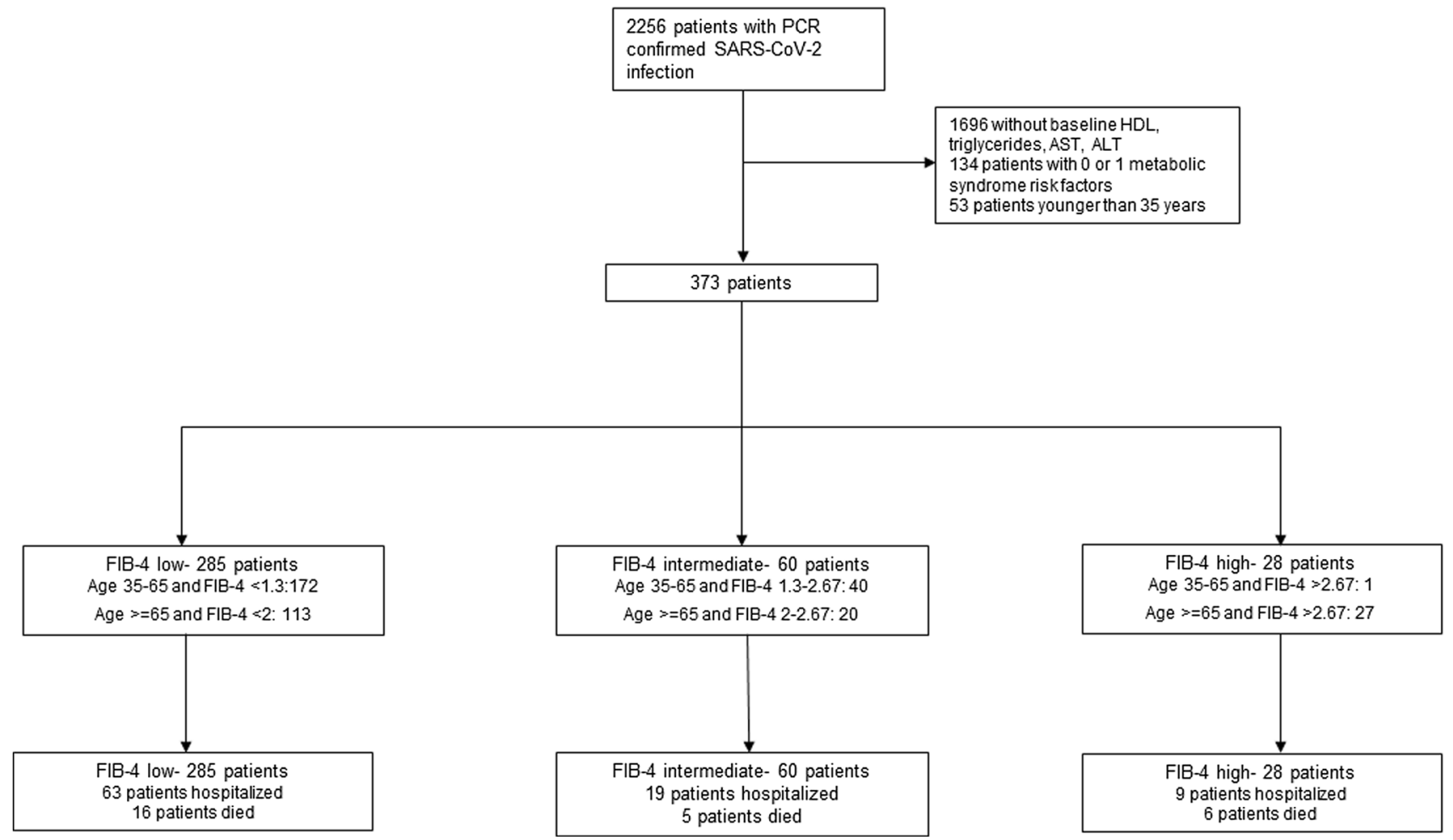

Fig. 1 Patient selection flow chart

these models and our primary model (FIB-4). All analyses were performed on Stata v14 (College Station, Texas).

\section{Results}

A total of 373 patients were included in the final cohort (Fig. 1). The median age was 62 years [Interquartile range (IQR) 54-74], 194 (52\%) were male, and 50 (13.40\%) were Hispanic. A total of 91 (24.40\%) patients required hospitalization and 27 (7.24\%) died. The median number of metabolic risk factors was 3 (IQR 2-4), and the median FIB-4 index was 1.10 (IQR 0.78-1.61). Differences in baseline characteristics by FIB-4 level are presented in Table 1 . Patients in the highest category of FIB-4 index were older (median 81 years, IQR 76-88.5) and had higher proportions of CAD (57.14\%) and CKD (57.14\%).

\section{Hospitalization}

Patients with intermediate FIB-4 score had 63\% higher odds of hospitalization compared to those in the low category (odds ratio [OR] 1.63 (95\% CI 1.13-2.35); $p=0.01$ ) and those with a high FIB-4 index had 67\% higher odds (OR 1.67 (95\% CI 1.14-2.45); $p=0.01$ ) (Table 2). After adjusting for $\mathrm{DM}$ and $\mathrm{CKD}$, the estimates were largely unchanged for patients in the intermediate category having $67 \%$ higher odds of hospitalization (OR 1.67 (95\% CI 1.06-2.64); $p=0.03$ ) but an association was no longer seen for those with values $>2.67$ (OR 0.96; 95\% CI 0.84-1.10); $p=0.54$ ). Other variables associated with increased odds of hospitalization in our cohort were male sex (OR 1.48, 95\% CI $1.02-2.14 ; p=0.04)$ and CAD (OR 1.69, 95\% CI 1.14-2.50; $p=0.01$ ). Hispanic patients had $56 \%$ lower odds of hospitalization (OR 0.44 (95\% CI 0.26-0.75); $p=0.01$ ) compared to non-Hispanic patients.

\section{Mortality}

Patients with FIB-4 score $>2.67$ were found to have almost fivefold odds of in-hospital mortality compared to those in the low and intermediate categories, with an OR of 4.59 (95\% CI 1.46-14.44; $p=0.01$ ) (Table 2) but no association with intermediate FIB-4 index (OR 1.53 (95\% CI 0.34-6.94; $p=0.58$ ). After adjusting for DM and CKD, the effect of FIB-4 decreased, with an OR of 2.22 (95\% CI 1.20-4.12; $p=0.01)$ in the high-risk category. Higher odds of in-hospital mortality were also seen with increasing age (OR 1.08, 95\% CI $1.05-1.10 ; p<0.001)$, history of hypothyroidism (OR 1.82, 95\% CI 1.14-2.89; $p=0.01$ ), and low HDL at baseline (OR 7.82, 95\% CI 1.45-42.10; $p=0.02$ ). 
Table 1 Baseline demographic characteristics by FIB-4 score

\begin{tabular}{|c|c|c|c|c|c|}
\hline & $\begin{array}{l}\text { All } \\
N=373\end{array}$ & $\begin{array}{l}\text { Low }^{\dagger} \\
n=285\end{array}$ & $\begin{array}{l}\text { Intermediate }^{\dagger} \\
n=60\end{array}$ & $\begin{array}{l}\operatorname{High}^{\dagger} \\
n=28\end{array}$ & $p$ value \\
\hline Age (years) & $62(54-74)$ & $62(51-73)$ & $61(54-71.5)$ & $81(76-88.5)$ & 0.001 \\
\hline Male & $194(52 \%)$ & $148(51.9 \%)$ & $32(53.3 \%)$ & $14(50 \%)$ & 0.96 \\
\hline Hispanic & $50(13.4 \%)$ & $41(14.4 \%)$ & $8(13.3 \%)$ & $1(3.4 \%)$ & 0.28 \\
\hline Metabolic syndrome risk & $3(2-4)$ & $3(2-4)$ & $3(2-4)$ & $3(2-4)$ & 0.67 \\
\hline Diabetes mellitus type 2 & $134(35.9 \%)$ & $108(37.9 \%)$ & $18(30 \%)$ & $8(28.6 \%)$ & 0.36 \\
\hline Hypertension & $265(71 \%)$ & $197(69.1 \%)$ & $46(76.7 \%)$ & $22(78.6 \%)$ & 0.33 \\
\hline Hypertriglyceridemia & $179(48 \%)$ & $140(49.1 \%)$ & $28(46.7 \%)$ & $11(39.3 \%)$ & 0.60 \\
\hline Dyslipidemia & $292(78.3 \%)$ & $220(77.2 \%)$ & $46(76.7 \%)$ & $26(92.9 \%)$ & 0.15 \\
\hline Central obesity & $303(81.2 \%)$ & $234(82.1 \%)$ & $52(86.7 \%)$ & $17(60.7 \%)$ & 0.01 \\
\hline BMI category & & & & & $<0.001$ \\
\hline Less than $25 \mathrm{~kg} / \mathrm{m}^{2}$ & $51(13.7 \%)$ & $34(11.9 \%)$ & $6(10 \%)$ & $11(39.3 \%)$ & \\
\hline $25-29.9 \mathrm{~kg} / \mathrm{m}^{2}$ & $111(29.8 \%)$ & $78(27.4 \%)$ & $22(36.7 \%)$ & $11(39.3 \%)$ & \\
\hline $30 \mathrm{~kg} / \mathrm{m}^{2}$ or higher & $211(56.6 \%)$ & $173(60.7 \%)$ & $32(53.3 \%)$ & $6(21.4 \%)$ & \\
\hline Coronary artery disease & $80(21.4 \%)$ & $54(18.9 \%)$ & $10(16.7 \%)$ & $16(57.1 \%)$ & $<0.001$ \\
\hline Chronic kidney disease & $65(17.4 \%)$ & $40(14 \%)$ & $9(15 \%)$ & $16(57.1 \%)$ & $<0.001$ \\
\hline Hypothyroidism & $53(14.2 \%)$ & $39(13.7 \%)$ & $10(16.7 \%)$ & $4(14.3 \%)$ & 0.83 \\
\hline Obstructive sleep apnea & $67(18 \%)$ & $49(17.2 \%)$ & $14(23.3 \%)$ & $4(14.3 \%)$ & 0.46 \\
\hline Baseline AST (Units/Liter) & $21(17-29)$ & $20(15-27)$ & $31(23-42.5)$ & $25.5(19-40)$ & $<0.001$ \\
\hline Baseline ALT (Units/Liter) & $26(18-38)$ & $26(18-38)$ & $29.5(23-46.5)$ & $16.5(11.5-23.5)$ & 0.002 \\
\hline Baseline platelet count $\left(\times 10^{9}\right.$ per liter $)$ & $236(188-288)$ & $252(209-301)$ & $181(139.5-243)$ & $151.5(130-182)$ & 0.001 \\
\hline Region $^{\dagger \dagger}$ & & & & & 0.06 \\
\hline Region 1 & $130(31.2 \%)$ & $89(31.2 \%)$ & $25(41.7 \%)$ & $16(57.1 \%)$ & \\
\hline Region 2 & $69(18.5 \%)$ & $62(21.8 \%)$ & $5(8.3 \%)$ & $2(7.1 \%)$ & \\
\hline Region 3 & $1(0.3 \%)$ & 0 & $1(1.7 \%)$ & 0 & \\
\hline Region 4 & $15(4 \%)$ & $12(4.2 \%)$ & $2(3.3 \%)$ & $1(3.6 \%)$ & \\
\hline Region 5 & $25(6.7 \%)$ & $20(7 \%)$ & $5(8.3 \%)$ & 0 & \\
\hline Region 6 & $132(35.4 \%)$ & $101(35.4 \%)$ & $22(36.7 \%)$ & $9(32.1 \%)$ & \\
\hline Region 7 & $1(0.3 \%)$ & $1(0.3 \%)$ & 0 & 0 & \\
\hline
\end{tabular}

Median (IQR) or n (\%); $p$ values from Kruskal-Wallis test for continuous variables, Chi-squared test for categorical variables (or Fisher's Exact test if any cell sizes $<5$ )

${ }^{\dagger}$ FIB-4 categories, age 35-65: low $<1.30$, intermediate 1.30-2.67 and high $>2,67$; age 65 and older: low $<2$, intermediate 2-2.67, high $>2.67$

${ }^{\dagger \dagger}$ See Supplemental Fig. 1

\section{Age and AST/ALT ratio}

Our final model with FIB-4 as the only independent variable had a c-statistic of 0.548 for hospitalization and 0.604 for mortality. When adjusted for CKD and DM these increased to 0.630 and 0.677 , respectively. CVD was considered to be on a causal pathway. Male sex, Hispanic race, hypothyroidism and low HDL at baseline did not meet criteria for confounding therefore we made no adjustment for these variables. Models with age as the only independent variable had a c-statistic of 0.570 for hospitalization and 0.788 for mortality, and with AST/ALT ratio as the only independent variable, 0.550 for hospitalization and 0.658 for mortality. Age and AST/ALT ratio combined gave c-statistics for hospitalization and mortality of 0.579 and 0.789 , respectively. With adjustment for CKD, these increased to 0.609 and 0.795 .

\section{Discussion}

In this study, we demonstrated that, among patients at risk of NAFLD, baseline FIB-4 index elevation prior to COVID-19 was associated with increased odds of hospitalization and inhospital mortality compared to those with low FIB-4. This association remained significant after adjusting for DM and CKD. However, when we evaluated the components of age and AST/ALT ratio in FIB-4 we found that models only 
Table 2 Crude and adjusted estimates of the effect of baseline FIB-4 score on hospitalization and in-hospital mortality among patients at risk of Non-Alcoholic Fatty Liver Disease diagnosed with COVID-19 $(N=373)$

\begin{tabular}{lllllll}
\hline & Hospitalized & Hospitalization & $p$ value & Died & In-hospital mortality & $p$ value \\
\hline $\begin{array}{l}\text { Crude effect } \\
\text { FIB-4 score* }\end{array}$ & & & & & \\
$\quad$ Low & $63 / 285$ & 1.00 (reference) & & $16 / 285$ & 1.00 (reference) & \\
$\quad$ Intermediate & $19 / 60$ & $1.63(1.13-2.35)$ & 0.01 & $5 / 60$ & $1.53(0.34-6.94)$ & 0.58 \\
$\quad$ High & $9 / 28$ & $1.67(1.14-2.45)$ & 0.01 & $6 / 28$ & $4.59(1.46-14.44)$ & 0.01 \\
Effect adjusted for DM2 and CKD ** & & & & & \\
FIB-4 score* & & & & & \\
$\quad$ Low & $63 / 285$ & $1.00($ reference) & & $16 / 285$ & 1.00 (reference) & \\
$\quad$ Intermediate & $19 / 60$ & $1.67(1.06-2.64)$ & 0.03 & $5 / 60$ & $1.52(0.37-6.34)$ & 0.56 \\
High & $9 / 28$ & $0.96(0.84-1.10)$ & 0.54 & $6 / 28$ & $2.22(1.20-4.12)$ & 0.01 \\
\hline
\end{tabular}

*For patients between age 35 years old and less than 65 , cutoffs were $<1.30,1.30-2.67$ and $>2.67$ for low, intermediate and high risk of fibrosis, respectively. For patients 65 years and older, the cutoffs used were $<2,2-2.67$ and $>2.76$ for low, intermediate and high risk of fibrosis, respectively

**Co-variate selection based on direct acyclic graphs presented in Supplemental Figure 2 with exact age were better at predicting hospitalization and mortality.

NAFLD is usually present as a clinically silent disease among patients with Type 2 DM and obesity and is often discovered incidentally during abdominal imaging performed for other indications. These two major MetS risk factors can precipitate and drive NAFLD progression to the aggressive non-alcoholic steatohepatitis (NASH) phenotype and advanced fibrosis if not controlled. Furthermore, MetS represent an ongoing pro-inflammatory state that is associated with excess of circulating cytokines and attenuation of body's immune response. In the setting of acute COVID-19 illness, the direct cytopathic effect of SARS CoV-2 in addition to underlying advanced liver fibrosis might exacerbate the virus-induced cytokine storm, possibly through hepatic release of proinflammatory cytokines [17]. While it is quite challenging to discern the independent contributions of the direct cytotoxic effect of SARS CoV-2 or the degree of underlying liver fibrosis on the poor clinical outcomes seen in patients with underlying risk factors for MetS such as those in our population, an adjunctive effect of underlying fibrosis and direct cytotoxic effect seems plausible.

Our findings are consistent with Targher et al. [18], who reported that intermediate and elevated FIB-4 on admission was associated with severe COVID-19 illness (unadjusted OR 4.32, 95\% CI 1.94 - 9.59) and (unadjusted OR 5.73, 95\% CI 1.84-17.90), respectively, among patients with imaging-based NAFLD. This association remained evident after adjusting for sex, obesity and diabetes if intermediate and elevated FIB-4 were combined. However, no adjustment for age was done. The findings of Targher et al. likely reflect some of the direct cytopathic effect of SARS-CoV-2 rather than the severity of underlying liver disease (as they relied on laboratory data drawn while individuals were acutely infected with SARS-CoV-2).
Although we found that FIB-4 index prior to acute illness can serve as a predictor of poor COVID-19 clinical outcomes among patients at risk of NAFLD, further analysis indicated that even in this high-risk population, age alone was the best predictor of hospitalization and mortality. This reflects the very strong age-dependent survival rates for COVID-19 [19].

Previous studies have also suggested a role for the FIB-4 score in predicting poor clinical outcomes regardless of underlying risk factors for NAFLD although adjustment for age has not always been carried out. In a recent multicenter study, lbanez-Samaniego et al. [20] reported that, among 160 middle-aged patients hospitalized with acute COVID-19, FIB-4 $\geq 2.67$ calculated from baseline laboratory data drawn on admission was positively associated with a greater need for intensive care and mechanical ventilatory support (OR 3.41, 95\% CI 1.30 - 8.92). Similarly, Sterling et al. [21] reported in a single-center retrospective study of 256 hospitalized patients with acute COVID-19 that FIB-4 $\geq 2.67$ was independently associated with the need for mechanical ventilation (OR 3.09; $95 \%$ CI 1.38-6.93) and increased 30-day mortality (HR 1.68; 95\% CI 1.19-2.38: $p=0.003$ ). These two studies did not adjust for age. A pre-published study by Rentsch et al. [22] reported that, among 297 patients hospitalized with acute COVID-19, 122 (21\%) required intensive care. After adjustment for significant clinical risk factors (DM, CKD, cardiovascular/respiratory disease and age), FIB-4 $>3.25$ had an adjusted OR of 8.73 (95\% CI 4.11-18.56) for hospitalization and OR 8.40 (95\% CI 2.90-24.28) for intensive care compared to those with FIB- $4<1.45$.

More recently, Sterling et al. in a large cohort of 4901 COVID-19 patients estimated twofold odds [OR $2.20(95 \%$ CI 1.40-3.46)] for mechanical ventilation using FIB-4 dichotomized at 3.04 [23]. This is similar to the effect 
that we estimated for mortality in our highest risk FIB-4 group (Sterling et al. did not report mortality). Our study had few patients $(n=20)$ with a FIB-4 score $>3.04$, and our findings suggest that moving our high-risk threshold would have made little or no difference. Indeed, Sterling et al. found similar area-under-the-curve for cut-points in the range 2.15-3.25 [23].

Our study has several limitations. There was limited ethnic diversity among our patient population as the majority of patients in our cohort were non-Hispanic Caucasians. Our cohort comprised patients considered to be at increased risk of NAFLD. We lacked objective data for NAFLD diagnosis because we were unable to confirm NAFLD either by imaging or biopsy due to the retrospective design of our study. Finally, despite this being one of the largest cohorts applying the FIB-4 index to patients with COVID-19, the sample was of modest size.

In conclusion, although the FIB-4 index is a simple pointof-care tool that can be applied by frontline providers to identify patients with metabolic syndrome risk factors and COVID-19 who are at higher risk of hospitalization or death, among patients with a high number of comorbidities, age alone is a stronger predictor of hospitalization and mortality. Larger prospective cohort studies with imaging or biopsyproven NAFLD are needed to examine the complex interplay between NAFLD and severity of acute COVID-19 illness and to improve the application of FIB-4 index beyond that provided by age alone.

Supplementary Information The online version contains supplementary material available at https://doi.org/10.1007/s10620-021-07120-0.

\section{Funding None.}

\section{Declarations}

Conflict of interest The authors declare they have no conflict of interest.

Ethical approval This study was approved by the Institutional Review Board of UnityPoint Health Des Moines.

\section{References}

1. Guan WJ, Ni ZY, Hu Y et al. Clinical characteristics of coronavirus disease 2019 in China. N Engl J Med. 2020;382:1708-1720.

2. World Health Organization. WHO Director-General's opening remarks at the media briefing on COVID-19-11 March 2020 Geneva 2020. Available at: https://www.who.int/dg/speeches/ detail/who-director-general-s-opening-remarks-at-the-media-brief ing-on-covid-19---11-march-2020. Accessed September 28, 2020.

3. Johns Hopkins Coronavirus Resources Center. COVID-19 Unites States Cases 2020. COVID-19 Unites States Cases. 2020. Available at: https://coronavirus.jhu.edu/us-map. Accessed January 31, 2021.
4. Koff WC, Williams MA. Covid-19 and immunity in aging populations - a new research agenda. N Engl J Med. 2020;383:804-805.

5. Ji D, Qin E, Xu J et al. Non-alcoholic fatty liver diseases in patients with COVID-19: a retrospective study. J Hepatol. 2020;73:451-453.

6. Richardson S, Hirsch JS, Narasimhan M, Crawford JM, McGinn T, Davidson KW et al. Presenting characteristics, comorbidities, and outcomes among 5700 patients hospitalized with COVID-19 in the New York City Area. JAMA. 2020;323:2052-2059.

7. Gao F, Zheng KI, Wang XB et al. Metabolic associated fatty liver disease increases coronavirus disease 2019 disease severity in nondiabetic patients. J Gastroenterol Hepatol. 2021;36:204-207.

8. Moore JX, Chaudhary N, Akinyemiju T. Metabolic syndrome prevalence by race/ethnicity and sex in the United States, National Health and Nutrition Examination Survey, 1988-2012. Prev Chronic Dis. 2017; 14:E24.

9. Eslam M, Sanyal AJ, George J. MAFLD: a consensus-driven proposed nomenclature for metabolic associated fatty liver disease. Gastroenterology. 2020;158:1999-2014.e1.

10. Eslam M, Newsome PN, Sarin SK, Anstee QM, Targher G, Romero-Gomez M et al. A new definition for metabolic dysfunction-associated fatty liver disease: an international expert consensus statement. J Hepatol. 2020;73:202-209.

11. Taylor RS, Taylor RJ, Bayliss S et al. Association between fibrosis stage and outcomes of patients with nonalcoholic fatty liver disease: a systematic review and meta-analysis. Gastroenterology. 2020;158:1611-25.e12.

12. Huang R, Zhu L, Wang J, Xue L, Liu L, Yan X et al. Clinical features of COVID-19 patients with non-alcoholic fatty liver disease. Hepatol Commun. 2020;4:1758-1768.

13. Sterling RK, Lissen E, Clumeck N, Sola R, Correa MC, Montaner $\mathrm{J}$ et al. Development of a simple noninvasive index to predict significant fibrosis in patients with HIV/HCV coinfection. Hepatology. 2006;43:1317-1325.

14. McPherson S, Hardy T, Dufour JF et al. Age as a confounding factor for the accurate non-invasive diagnosis of advanced NAFLD fibrosis. Am J Gastroenterol. 2017;112:740-751.

15. Bozeman SR, Hoaglin DC, Burton TM, Pashos CL, Ben-Joseph $\mathrm{RH}$, Hollenbeak CS. Predicting waist circumference from body mass index. BMC Med Res Methodol. 2012;12:115.

16. Hernán MA, Hernández-Díaz S, Werler MM, Mitchell AA. Causal knowledge as a prerequisite for confounding evaluation: an application to birth defects epidemiology. Am J Epidemiol. 2002; 155:176-184.

17. Portincasa P, Krawczyk M, Smyk W, Lammert F, Di Ciaula A. COVID-19 and non-alcoholic fatty liver disease: two intersecting pandemics. Eur J Clin Invest. 2020;50:e13338.

18. Targher G, Mantovani A, Byrne CD et al. Risk of severe illness from COVID-19 in patients with metabolic dysfunctionassociated fatty liver disease and increased fibrosis scores. Gut. 2020;69:1545-1547.

19. Centers for Disease Control and Prevention. COVID-19 Hospitalization and Death by Age 2020. Available at https://www.cdc. gov/coronavirus/2019-ncov/covid-data/investigations-discovery/ hospitalization-death-by-age.html.Accessed November 1, 2020.

20. Ibáñez-Samaniego L, Bighelli F, Usón C et al. Elevation of liver fibrosis index FIB-4 Is associated with poor clinical outcomes in patients with COVID-19. J Infect Dis. 2020;222:726-733.

21. Sterling RK, Oakes T, Gal TS, Stevens MP, deWit M, Sanyal AJ. The FIB-4 index is associated with need for mechanical ventilation and 30-day mortality in patients admitted with COVID-19. $J$ Infect Dis. 2020;222:1794-1797.

22. Rentsch CT, Kidwai-Khan F, Tate JP, et al. Covid-19 testing, hospital admission, and intensive care among 2,026,227 United States veterans aged 54-75 years. medRxiv. 2020:2020.04.09.20059964. 
23. Sterling RK, Shin D, Shin Y, et al. FIB-4 Predicts Need for Mechanical Ventilation in a Multi-ethnic National cohort of COVID-19. Hepatology Communications. Published online 29 April 2021.
Publisher's Note Springer Nature remains neutral with regard to jurisdictional claims in published maps and institutional affiliations. 